\title{
Capacitação em Epidemiologia na Perspectiva de Implantação do SUS: Integração Universidade e Serviços de Saúde - Campinas $1992-1994^{*}$
}

\section{Ana Maria Segall Corrêa **}

\section{Apresentação}

Esta apresentação refere-se a experiência em capacitação em epidemiologia de profissionais de saúde (DMPS, 1994), desenvolvida entre 1992 e 1994, pela área de epidemiologia do Departamento de Medicina Preventiva e Social, da Faculdade de Ciências Médicas da UNICAMP, como contribuição à implantação do SUS (Brasil, 1988), na região de Campinas. Suas atividades decorrem de convênio com o Centro Nacional de Epidemiologia do Ministério da Saúde e são orientadas pelas diretrizes (Ministério da Saúde, 1993) de descentralização e municipalização das ações e serviços de saúde. Como estratégias para a realização dos objetivos propostos, constituiu-se uma Comissão Inter-institucional de Acompanhamento e Avaliação (C.I.A.A.), ampliou-se o Laboratório de Aplicação em Epidemiologia ( $L A P E$ ), e desenvolveu-se metodologia de avaliação das atividades propostas. Foram realizados 23 cursos e 6 estágios, com participação de 389 profissionais de 22 municípios da região.

\footnotetext{
* AUTORES: SEGALL CORREA, A.M .; GONÇALVES, N.N.S.; STENZEL, A.C.; HOEHNE, E.J.; OLIVEIRA, H.B., do Depto de Medicina Preventiva e Social da UNICAMP e ROSSI, E., da Comissão de Pesquisa da Faculdade de Ciências Médicas da UNICAMP..

** Professora do Depto de Medicina Preventiva e Social da FCM/UNICAMP
} 


\section{Comissão Interinstitucional de Acompanhamento e Avaliação (C.I.A.A.)}

A Comissão Interinstitucional de Acompanhamento e Avaliação (C.I.A.A.), foi incumbida de dar, ao arcabouço geral do Convênio do CENEPI com a Unicamp, a feição peculiar da realidade local da região de Campinas, acompanhar e avaliar seu desempenho.

Participaram da C.I.A.A., profissionais de saúde das instituições regionais (8 ERSAs), de cinco Secretarias Municipais de Saúde, representantes do Centro de Vigilância Epidemiológica da Secretaria Estadual de Saúde (CVE), representantes do Hospital das Clínicas da UNICAMP e docentes da Área de Epidemiologia do DMPS. Os trabalhos desta comissão permitiram integração entre a Universidade e os Serviços de Saúde da região, fundamental para o desenvolvimento de todas as atividades, tendo atuado como importante referencial, para condução dos trabalhos em acordo com as realidades locais e regionais.

\section{Ampliação e organização do Laboratório de Aplícação em Epidemiologia (LAPE)}

O LAPE, criado 1991, através de apoio da Faculdade de Ciências Médicas da UNICAMP e do CNPq, com o objetivo entre outros, de desenvolver na área de epidemiologia do DMPS, atividades voltadas às necessidades das instituições de saúde da região, foi substancialmente ampliado com os recursos oriundos do convênio CENEPI/DMPS. Viabilizou-se, assim, o desenvolvimento das atividades de capacitação previstas em cursos e estágios, com práticas de manejo de informações epidemiológicas, além de suporte de informática para as assessorias a municípios instituições universitárias (Unicamp e outras universidades do país) e a diversos estágios informais.

\section{Atividades de capacitação}

Para a realização das atividades propostas, optou-se por metodologia de capacitação baseada, fundamentalmente, nos aspectos operacionais da epidemiologia, o que permitiu atender, pelo menos enquanto oferta de oportunidade, as necessidades de aquisição de habilidades para formulação de: i) propostas de mecanismos de controle de doenças; ii) elaboração de projeto e execução de investigação para diagnóstico de saúde; iii) elaboração de projeto e execução de avaliação das atividades de Vigilância Epidemiológica e avaliação de serviços de saúde.

Foram realizados 23 cursos de extensão e seis estágios em serviço com um total de 2.152 horas de capacitação, para profissionais dos serviços de saúde da região de influência de Campinas. Participaram destas atividades profissio- 
nais de 22 municípios e de 8 ERSAs da região. Os médicos e enfermeiros formaram constituíram a clientela predominante $(62 \%)$ deste programa.

\section{Processo de diagnóstico, acompanhamento e avaliação}

A necessidade sentida de avaliar o processo de capacitação desencadeado, bem como a necessidade de apontar novos rumos e diretrizes desta atividade, orientam o planejamento de instrumentos para: i) avaliação dos cursos pelos seus respectivos alunos; ii) análise de possíveis impactos destas ações de treinamento nas atividades destes profissionais nos serviços de saúde; iii) finalmente, para a análise das condições locais do uso do instrumental epidemiológico e conseqüente potencialidade de absorção dos conhecimentos, tecnologias e práticas epidemiológicas. Neste sentido, foram desenvolvidos dois questionários auto-aplicados, que, após discussão e reformulação pelos integrantes da C.I.A.A., foram pré-testados e posteriormente aplicados. O primeiro deles, respondidos pelos alunos, ao final de cada curso realizado, o segundo, após seis meses da conclusão dos mesmos, para avaliação de impacto da capacitação sobre as atividades profissionais dos ex-alunos e, por fim um terceiro instrumento, para diagnóstico do uso do instrumental epidemiológico, avaliação dos recursos humanos e materiais disponíveis nos serviços de saúde, da região de Campinas.

Os questionários de avaliação dos conteúdos didáticos pedagógicos foram distribuídos ao final de 20 dos 23 cursos oferecidos. Foram analisados 264 questionários dos 309 distribuídos aos alunos, o que representa 85,4 daqueles que tiveram oportunidade de participar da avaliação.

Mais de $90 \%$ dos alunos, refere como excelente ou boa, a proposta de conteúdos, formato e metodologia didático-pedagógica dos cursos. Também, mais de $90 \%$ dos profissionais disseram que foi grande, o interesse despertado pelos temas abordados. A participação dos docentes foi globalmente avaliada como boa ou excelente por $94,8 \%$ dos alunos.

No segundo componente, avaliação de impacto, procurou-se conhecer melhor as características profissionais dos alunos, sua área de atuação e mobilidade funcional. Relativamente às atividades de capacitação oferecidas, buscou-se conhecer sua contribuição tanto no aspecto mais geral (se contribui ou não), quanto no aspecto qualitativo (qual seria a característica desta contribuição) e também, o papel de apoio ou de restrição institucional às atividades profissionais dos ex-alunos.

Dos questionários enviados por correio, juntamente com um envelope selado para postagem de retorno, obteve-se $52,6 \%$ de resposta. A maioria dos profissionais desenvolvia atividades na área de vigilância epidemiológica $(45,1 \%)$, seguidos de $11,3 \%$ cujas atividades eram em vigilância sanitária. Apenas $4,2 \%$ exerciam integralmente vigilância sanitária e epidemiológica. 
Estas proporções, relativas às áreas de vigilância $(60,6 \%)$, mostram que as atividades de capacitação foram predominantemente dirigidas aos profissionais que, em algum nível, manejam informações epidemiológicas. Do total, 22,5\% eram profissionais ligados a atividades assistenciais, entretanto, considerando o contato mantido com os alunos, percebe-se que muitos estavam ligados a programas de controle de doenças e consequentemente, conviviam em seu trabalho, com a necessidade de conhecimentos específicos em epidemiologia.

A rotatividade dos profissionais de saúde não foi problema nestes primeiros 6 meses após curso, $79 \%$ destes, mantinham as mesmas atividades que exerciam no período anterior ao curso. Daqueles que tiveram suas atribuições alteradas, 8(61\%) passaram a exercer funções epidemiológicas mais complexas.

Os cursos e estágio trouxeram contribuição significativa para o exercício profissional de $70,5 \%$ dos entrevistados. Considerando os que mencionavam contribuição parcial às atividades, esta proporção sobe para $87,3 \%$. $\mathrm{Na}$ opinião de 17 ex-alunos (24\% do total), não houve aproveitamento adequado dos conteúdos dos cursos e estágios nas atividades em serviço. Na maioria das vezes $(64,7 \%)$, isto ocorreu devido às dificuldades ligadas às condições de trabalho $e$ a alguns fatores político-institucionais.

Ao perguntar sobre as características do impacto das atividades de capacitação, no desempenho profissional dos alunos, obtivemos 88 respostas, das quais $63,6 \%$ foram referências de melhoria na qualidade do trabalho e aumento de responsabilidade profissional, $26 \%$ dos pesquisados apontaram acréscimos de atividades, conseqüente à capacitação. Com referência à percepção do suporte que as instituições oferecem aos seus profissionais recém capacitados, 80,3 sentem-se parcial $(54,9 \%)$ ou totalmente $(25,4 \%)$ apoiados política e administrativamente. Entretanto, 15,5\% referem total ausência de apoio.

Com relação às perspectivas futuras, foi solicitado ao profissional a referência das condições que poderiam implicar em melhoria de seu desempenho profissional. As respostas que apareceram com mais freqüência foram: educação continuada/assessoria em serviço $(22,4 \%)$, definição de política adequada de recursos humanos $(17,2 \%)$, condições para informatização das atividades de vigilância $(18,5 \%)$, políticas específicas para Vigilância à Saúde $(18,5 \%)$ e, finalmente, recursos materiais e área física adequada, que foi referido por $11,2 \%$ dos profissionais. Todos os profissionais referem de alguma forma à necessidade de política de capacitação de RH, que contemple as atividades de acompanhamento, reciclagem, assessoria e supervisão, todas elas voltadas para atuação em serviço e oferecidas de forma contínua. Esta avaliação do impacto das atividades de capacitação terá continuidade com o envio do questionário aos demais profissionais treinados, obedecendo a mesma sistemática utilizada até aqui.

No terceiro componente do processo de avaliação, buscou-se identificar e analisar o ambiente político-institucional, as características das atividades rela- 
tivas à Epidemiologia, como instrumento de trabalho nos diversos níveis dos serviços de saúde (municipais e regionais), bem como o perfil dos recursos humanos existentes e recursos materiais disponíveis.

De fato, são freqüentes, entre os profissionais da área de saúde, as discussões sobre a necessidade de uma nova prática epidemiológica nos serviços de saúde. Isto implica na implementação de modelo de vigilância (PAIM, 1993) à saúde que incorpore, de forma integrada e compatível com as necessidades e realidades locais, as tradicionais atividades de vigilância epidemiológica, sanitária, controle de vetores e vigilância de riscos ambientais. $\mathrm{O}$ conhecimento destas práticas, dos recursos humanos envolvidos, das estruturas institucionais e materiais disponíveis são pré-condições para a orientação do planejamento, tanto de implementação desta nova abordagem da epidemiologia em serviços de saúde, como da capacitação dos recursos humanos necessários.

Os dados coletados em 42 dos 85 municípios da região de Campinas estão ainda em fase de análise. Dados preliminares mostram que de um modo geral as condições estruturais são propícias para melhor desenvolvimento das práticas epidemiológicas em nível local. Nota-se, entretanto, uma centralização muito grande da vigilância epidemiológica, falta de integração com as demais atividades de vigilância e sobretudo ausência de utilização dos recursos da epidemiologia para a definição de diretrizes assistenciais e planejamento das ações de saúde.

\section{Conclusões}

O trabalho desenvolvido pela área de epidemiologia do DMPS, no âmbito do convênio Ministério da Saúde - CENEPI/Unicamp - DMPS, ultrapassou as expectivas iniciais, naquilo que se refere à capacitação de recursos humanos. Várias circunstâncias são responsáveis por este fato. Destaca-se, concomitantemente ao treinamento realizado a procura de estabelecimento de condições de integração com as diversas instituições que compõem o SUS da região, conhecimento das características básicas dos serviços de saúde, especialmente quanto a capacidade e potencialidades de desenvolvimento de atividades de epidemiologia.

O processo de avaliação e diagnóstico possibilitaram à universidade, às instituições locais e regionais de saúde e ao próprio CENEPI, referências pelas quais os futuros compromissos possam ser balizados. As informações obtidas também sugerem que, tanto as atividades de capacitação, quanto os componentes de diagnóstico e avaliação repercutiram de forma positiva nas atividades dos profissionais de saúde envolvidos neste esforço.

A experiência até aqui relatada colocam ainda, para discussão, o papel e a contribuição da Universidade, sua relação com as instituições prestadoras de 
serviço de saúde (Brasil, 1993b) e seus órgãos específicos de desenvolvimento de recursos humanos. Tanto os dados obtidos através da busca de opinião dos alunos, quanto a vivência com eles ensinaram que é necessário buscar efetividade nas ações de capacitação, através de um trabalho complementar e continuado que poderá ser desempenhado pelas universidades. Estas devem (ABRASCO, 1989) estimular e apoiar os órgãos de recursos humanos das instituições de saúde, suprir suas necessidades com programas e conteúdos específicos de epidemiologia, principalmente aqueles não desenvolvidos rotineiramente nos serviços.

\section{Referências Bibliográficas}

ABRASCO Plano diretor para o desenvolvimento da epidemiologia no Brasil. Rio de Janeiro, s.d. [Proposta elaborada no seminário "Estratégias para o Desenvolvimento da Epidemiologia no Brasil", realizado em Itaparica, BA, maio de 1989].

BRASIL - Constituição da República Federativa do Brasil, 1988. Brasília. Senado Federal, 1988. Artigo 200 - Inciso II, Artigo 201 - Inciso I.

MINISTÉRIO DA SAÚDE. Descentralização das ações e serviços de saúde: a ousadia de cumprir e fazer cumprir a lei. Brasília: Ministério da Saúde, 1993.

MINISTÉRIO DA SAÚDE $A$ investigação sobre recursos humanos em saúde. Brasília, Ministério da Saúde, $1993 b$.

PAIM, J.S. \& TEIXEIRA, M.G.L.C. Reorganização do sistema de vigilância epidemiologica na Perspectiva do Sistema Único de Saúde (SUS). Brasília CENEPI., 1993. (Relatório interno do Ministério da Saúde).

UNIVERSIDADE ESTADUAL DE CAMPINAS (UNICAMP). Departamento de Medicina Preventiva e Social. Capacitação em epidemiologia para o SUS. Relatório de atividades, Campinas, 1994. 